\title{
Publisher's Note: Theory of two-dimensional spatially indirect equilibrium exciton condensates [Phys. Rev. B 92, 165121 (2015)]
}

Feng-Cheng Wu, Fei Xue, and A. H. MacDonald

(Received 17 November 2015; published 3 December 2015)

DOI: 10.1103/PhysRevB.92.239901

PACS number(s): 73.21.-b, 71.35.-y, 99.10.Fg

This paper was published online on 21 October 2015 with a typographical error in Eq. (20). Equation (20) should read as

$$
\mathcal{Z}=\sqrt{1-\sum_{\vec{k}, \vec{Q}}\left|z_{\vec{k}}(\vec{Q})\right|^{2}}
$$

The equation has been corrected as of 16 November 2015. The equation is incorrect in the printed version of the journal. 